\title{
Effects of metformin on clinical outcomes in patients with type 2 diabetes and COVID-19
}

Nasser Mikhail, $\mathrm{MD}^{1^{*}}$, Soma Wali, $\mathrm{MD}^{2}$

${ }^{1}$ Endocrinology Division, Department of Medicine, Olive-View-UCLA Medical Center, David-Geffen School of Medicine, CA, USA. ${ }^{2}$ Department of Medicine, Olive-View-UCLA Medical Center, David-Geffen School of Medicine, CA, USA.

*Corresponding author: Nasser Mikhail, Endocrinology Division, Department of Medicine, Olive-View-UCLA Medical Center, DavidGeffen School of Medicine, CA, USA.

Received Date: April 21, 2021; Accepted Date: April 28, 2021; Published Date: May $12,2021$.

Citation: N Mikhail, S Wali. (2021) Effects of metformin on clinical outcomes in patients with type 2 diabetes and COVID-19. Journal of Endocrinology and Disorders. 5(3): Doi: 10.31579/2640-1045/072

Copyright: () 2021 Nasser Mikhail. This is an open-access article distributed under the terms of the Creative Commons Attribution License, which permits unrestricted use, distribution, and reproduction in any medium, provided the original author and source are credited.

\begin{abstract}
Background: The effects of metformin therapy on the prognosis of patients with coronavirus disease 2019 (COVID-19) are unclear.
\end{abstract}

Objective: To review effects of metformin on clinical outcomes, particularly mortality, in patients with type 2 diabetes and COVID-19.

Methods: Review of English literature by PUBMED search until April 20, 2021. Search terms included diabetes, COVID-19, metformin, Retrospective studies, meta-analyses, pertinent reviews, and consensus guidelines are reviewed.

Results: All available studies in this area are retrospective. Two population-based studies did not find significant association between metformin use and susceptibility to COVID-19. Most, but not all studies, suggest that metformin use prior to hospital admission might be associated with significant decrease in mortality in patients with type 2 diabetes and COVID-19. Continuing metformin use after hospital admission did not have significant impact on COVID-19 related death but may decrease risk of acute respiratory distress syndrome (ARDS). Meanwhile, in-hospital metformin administration may be associated with approximately 4.6 times increase risk of lactic acidosis in patients with severe symptoms of COVID-19, patients taking $\geq 2 \mathrm{gm} / \mathrm{d}$ of metformin, and patients with estimated glomerular filtration rate (eGFR) less than $60 \mathrm{ml} / \mathrm{min} / 1.73 \mathrm{~kg} / \mathrm{m}^{2}$.

Conclusions: Although most retrospective studies suggest that metformin administration may be associated with decreased risk of COVID-19 mortality, these data should be confirmed by randomized trials. In patients with type 2 diabetes and COVID-19 admitted to the hospital, metformin use should be avoided in presence of severe symptoms of COVID-19, kidney dysfunction (eGFR $<60 \mathrm{ml} / \mathrm{min} / 1.73 \mathrm{~m}^{2}$ ), and in daily doses of $\geq 2$ gm due to increased risk of lactic acidosis.

Key words: COVID-19; diabetes; metformin; safety; mortality; lactic acidosis

\section{Introduction}

Metformin is the most common anti-diabetic drug worldwide due to its well-established long-term overall high efficacy and safety profile and low cost [1]. The effects of metformin therapy on risk and clinical outcomes in COVID-19 are unclear. The American Diabetes Association (ADA) recommends insulin as the standard therapy for hyperglycemia in hospital [2]. Moreover, the ADA generally recommends stopping all oral anti-diabetic agents in most patients after hospital admission admitted to the hospital due to due to lack of data, presence of adverse effects, and limited efficacy [2]. Meanwhile, metformin might be an exception due to emerging studies showing potential benefits in patients with COVID-19.
This review discusses the effects of metformin on susceptibility and prognosis of COVID-19 with special emphasis on its impact on mortality.

\section{Effect of metformin on susceptibility to COVID-19}

Two large studies investigated risk of COVID-19 in relation to prior metformin use with different results [3,4]. In a large population-based study from South Korea, Oh et al [3] performed propensity score matching analysis to compare risk of COVID-19 between patients with type 2 diabetes prescribed metformin $(n=5,946)$ with those not prescribed metformin $(5,946)$. They found that patients prescribed metformin had $30 \%$ lower risk of COVID-19 compared with those not prescribed metformin, odds ratio (OR) 0.70 (95\% CI 0.61-0.80; $\mathrm{P}<0.001$ ) [3]. However. in another larger propensity score matching analysis from the 
UK, Wang et al [4] found no significant difference in incidence of COVID-19 between the group of patients exposed to metformin $(n=10,183)$ and the matched group not exposed to metformin, adjusted hazard ratio (HR) 0.80 (95\% CI 0.49-1.30).

\section{Effect of metformin on mortality in patients with COVID-19}

\section{Effect of pre-admission use of metformin on mortality}

The largest study that examined association of metformin and other glucose-lowering therapies with mortality risk in patients with COVID19 was conducted by Khunti et al [5] in the UK. This was a nationwide population study including 2,851,465 subjects that represented almost the entire patients with type 2 diabetes in the UK [5]. Overall, COVID-19 related death occurred in $13,479(0.5 \%)$ subjects during the study period from February 16 to August $31^{\text {st }}, 2020$ [5]. After adjusting for differences in patients' characteristics, adjusted hazard ratio (HR) for COVID-19 related death was 0.77 (95\% CI, 0.73-0.81) for patients prescribed metformin compared with those not prescribed metformin [5]. Corresponding HR for sodium-glucose co-transporter 2 (SGLT2) inhibitors was 0.82 (95\% CI, 0.74-0.91) and for sulfonylureas was 0.94 (95\% CI 0.89-0.99) [5]. Risk of mortality was not statistically significant with thiazolidinediones HR 0.94 (95\% CI 0.82-1.07), and glucagon-like peptide 1 receptor agonists (GLP-1 RA) HR 0.94 (95\% CI 83-1.07) [5]. Interestingly, mortality risk was significantly increased with insulin therapy HR 1.43 (95\% CI 1.35-1.49) and to a lesser extent with dipeptidyl-peptidase-4 (DPP-4) inhibitors HR 1.07 (95\% CI 1.01-1.13) [5]. However, it should be emphasized that these results may be confounded by indication since metformin is usually prescribed in the early course of diabetes as opposed to insulin which is prescribed in more advanced stages of the disease. The CORONADO study is a French nationwide retrospective investigation that included 2,796 patients with type 2 diabetes admitted to the hospital with COVID-19 [6]. After adjusting for multiple variables, the authors found that metformin treatment before admission was significantly associated with lower risk of in-hospital death, OR 0.65 (95\% CI 0.45-0.93) and increased hospital discharge within 28 days OR 1.40 (95\% CI 1.08-1.81) [6]. In a metaanalysis conducted by Kow and Hasan [7], the authors analyzed data (up to August 8, 2020) of 5 studies including 8,121 patients with diabetes and COVID-19 who were using metformin prior to hospital admission. Their pooled analysis revealed a significantly decreased risk of mortality with the use of metformin prior to admission, pooled OR being 0.62 (95\% CI, 0.43-0.89) compared to patients with diabetes who were non-users of metformin [7]. Interestingly, the largest study included in this metaanalysis conducted by Bramante et al [8] showed that pre-admission metformin use was associated with decreased in-hospital mortality in women only, OR 0.78 (95\% CI 0.65-0.95), but not in men.

However, not all studies showed mortality reduction in patients with COVID-19 using metformin. In the propensity matched analysis by Wang et al [4] in the UK mentioned earlier, no significant difference in COVID19-related death was found between metformin users and non-users, adjusted HR 0.87 (95\% CI 0.34-2.20). Similarly, in the Korean study by Oh et al [3], metformin use was not associated with in-hospital mortality, adjusted HR 1.26 (95\% CI 0.81-1.95).

Taken together, the balance of evidence overall suggests that metformin use might be associated with mortality reduction among patients with type 2 diabetes and COVID-19. However, this conclusion should be considered preliminary due to the following limitations. First, all included studies were retrospective prone for confounding factors. Second, it was not possible to know to what extent patients were adherent to metformin intake prior to hospital admission. Third, it was not clear in any of these studies whether patients continued to take metformin or discontinued it after admission to the hospital.

\section{Effect of in-hospital administration of metformin on mortality}

In a relatively large $(n=1,213)$ study from China, Cheng et al [9] have shown that metformin use while in hospital was not associated with 28day all-cause mortality compared with metformin non-users; adjusted HR 0.87 (95\% CI 0.36-2.12; $\mathrm{P}=0.75)$. Similarly, in another smaller $(\mathrm{n}=328)$ retrospective Chinese study, Jiang et al [10] did not found significant association between metformin use and 30-day mortality. In a third small Chinese studies conducted by Cheng et al [11] including 50 patients with type 2 diabetes admitted for COVID-19, 22 patients continued to take metformin after admission. (median dose $1.0 \mathrm{~g} / \mathrm{d}$ ). Again, the authors found no difference in mortality or fever duration between metformin users and non-users in the hospital [11].

\section{Effects of metformin on other clinical outcomes of COVID- 19}

Ghany et al [12] conducted a retrospective study including 1,139 COVID19 positive Medicare elderly patients (mean age 71 years-old) across 8 states in the US. This cohort also included a majority of Black patients $(71 \%)$ and women $(60 \%)$ [12]. The authors found that metformin users $(n=392)$ had significantly lower rates of hospitalization compared to metformin non-users; adjusted relative hazard (RH) 0.71 (95\% CI 0.520.86) [12]. Furthermore, metformin users developed less frequently ARDS compared with non-users, adjusted RH 0.71 (95\% CI 0.52-0.86) [12]. In another retrospective study from China, Cheng et al [9] found that metformin in-hospital use was associated with decreased risk of heart failure adjusted HR 0.61 (95\% CI 0.43-0.87; P=0.006), and ARDS, adjusted HR $0.66,95 \%$ CI $0.46-0.96 ; \mathrm{P}=0.03$ ). There was no significant effect of metformin on acute kidney injury or disseminated intravascular coagulation [9]. Likewise, Jiang et al [10] found that COVID-19 patients with type 2 diabetes who received metformin during hospitalization had significantly lower risk of ARDS compared with non-metformin users after propensity score matching [adjusted OR 0.16 (95\% CI, 0.04-0.72; $\mathrm{P}=0.016)]$.

\section{Dose-dependent effects of metformin in COVID-19}

Two retrospective investigations addressed the impact of metformin doses on clinical outcomes in patients with type 2 diabetes COVID-19. In the first study of Medicare patients, Ghany et al [12] reported a doseeffect of metformin in terms of reduced risk of mortality. Thus, patients who were prescribed metformin $500-850 \mathrm{mg} / \mathrm{d}$ had HR of mortality of 0.35 (95\% CI 0.17-0.71), whereas those patients prescribed metformin at $\geq 1 \mathrm{~g} / \mathrm{d}$ had HR of mortality of 0.23 (95\% CI 0.06-0.78) [12]. Cheng et al [11] noted that the dose of metformin prior to admission showed a negative association with the rate of admission to intensive care unit with borderline statistical significance (OR 0.04, 95\% CI 0.00-0.99, $\mathrm{P}=0.049$ ).

\section{Safety of metformin use during hospitalization of patients with COVID-19}

Lactic acidosis is a rare, but potentially lethal adverse effect of metformin [13]. Cheng et al [9] evaluated in-hospital metformin safety in different subgroups of patients admitted with COVID-19 and type 2 diabetes. They found that lactic acidosis was increased among metformin users compared with metformin non-users, adjusted HR 4.66, 95\% 1.45-14.99; $\mathrm{P}=0.01$ [9]. Yet, this increased risk of lactic acidosis was limited to the following 
subgroups of patients: those with severe form of COVID-19, patients using metformin in doses of $\geq 2 \mathrm{~g} / \mathrm{d}$, and in presence of kidney dysfunction defined as eGFR $<60 \mathrm{ml} / \mathrm{min} / 1.73 \mathrm{~kg} / \mathrm{m}^{2}$ [9].

\section{Potential mechanisms underlying metformin clinical benefits}

Metformin has been shown to improve the immune response and reduce inflammation [14]. It may also block mTOR pathway which plays a role in the pathogenesis of Middle East respiratory syndrome coronavirus infection [15]. It should be emphasized, however, that metformin should not be used in presence of hypoxia, decreased tissue perfusion, sepsis, acute or chronic kidney disease, and acute heart and liver failure to avoid the risk of lactic acidosis [13].

\section{Conclusions and clinical implications}

Preliminary data suggest that metformin use prior to hospitalization of patients with type 2 diabetes and COVID-19 may reduce mortality. Meanwhile, continuing metformin after hospitalization did not affect mortality, but increased risk of lactic acidosis. The risk of lactic acidosis was evident only in patients with severe symptoms of COVID-19, those with kidney dysfunction, and in patients taking $\geq 2$ g of metformin daily. Accordingly, metformin should be discontinued in cases of severe COVID-19 and kidney disease, and its daily doses should not exceed $2 \mathrm{~g}$. On the other hand, metformin in lower doses may be continued in mild cases, and in presence of normal kidney function in view of its possible benefits in reducing risk of ARDS. Unfortunately, all current data related to COVID-19 and metformin are based on retrospective studies prone for multiple bias and confounding factors. Randomized trials are urgently needed to determine safety and efficacy of metformin in hospitalized COVID-19 patients. The COVIDOUT trial (NCT04510194) is a randomized trial that started January 2021 and expected to include 750 participants [16]. The purpose of this study is to see whether metformin treatment in non-hospitalized adults (30-85 years-old) with COVID-19 infection can prevent hypoxia, emergency room utilization, and COVID19 disease progression [16]. Interestingly, type 2 diabetes is not a requirement to participate in this study. Thus, the results of the COVIDOUT trial should clarify the potential benefits of metformin in patients with COVID-19 irrespective of presence of diabetes [16].

\section{Disclosure}

The authors do not have any conflict of interest to disclose.

\section{References}

1. American Diabetes Association. Pharmacologic approaches to glycemic treatment. Standards of Medical Care in diabetes2020; 43: S98-S110.

2. American Diabetes Association. Diabetes care in the hospital: Standards of Medical Care in diabetes-2020; 43: S193-S201.
3. Oh TK, Song IA. (2021) Metformin use and risk of COVID-19 among patients with type II diabetes mellitus: an NHISCOVID-19 database cohort study. Acta Diabetol. 13:1-8.

4. Wang J, Cooper JM, Gokhale K, Acosta-Mena D, Dhalla S, et al (2021) Association of metformin with susceptibility to COVID-19 in people with Type 2 diabetes. J Clin Endocrinol Metab. Epub ahead of print.

5. Khunti K, Knighton P, Zaccardi F, Bakhai C, Barron E, et al. (2021) Prescription of glucose-lowering therapies and risk of COVID-19 mortality in people with type 2 diabetes: a nationwide observational study in England. Lancet Diabetes Endocrinol. 9(5):293-303.

6. Wargny M, Potier L, Gourdy P, Pichelin M, Amadou C, et al. (2021) for the CORONADO investigators. Predictors of hospital discharge and mortality in patients with diabetes and COVID-19: updated results from the nationwide CORONADO study. Diabetologia. 64(4):778-794.

7. Kow CS, Hasan SS. (2021) Mortality risk with preadmission metformin use in patients with COVID-19 and diabetes: A meta-analysis. J Med Virol. 93(2):695-697.

8. Bramante CT, Ingraham NE, Murray TA, Marmor S, Hovertsen S, et al. (2021) Metformin and risk of mortality in patients hospitalised with COVID-19: a retrospective cohort analysis. Lancet Healthy Longev. 2(1):e34-e41.

9. Cheng X, Liu YM, Li H, Zhang X, Lei F, et al. (2020) Metformin is associated with higher incidence of acidosis, but not mortality, in individuals with COVID-19 and pre-existing type 2 diabetes. Cell Metab. 32(4):537-547.e3.

10. Jiang N, Chen Z, Liu L, Yin X, Yang H, et al. (2021) Association of metformin with mortality or ARDS in patients with COVID-19 and type 2 diabetes: A retrospective cohort study. Diabetes Res Clin Pract.173:108619.

11. Cheng X, Xin S, Chen Y, Li L, Chen W, Li W, Zhou B, Li C, Gong Y, Li F, Duan P, Zhou X. (2021) Effects of metformin, insulin on COVID-19 patients with pre-existed type 2 diabetes: A multicentral retrospective study. Life Sci.275:119371.

12. Ghany R, Palacio A, Dawkins E, Chen G, McCarter D et al. (2021) Metformin is associated with lower hospitalizations, mortality and severe coronavirus infection among elderly medicare minority patients in 8 states in USA. Diabetes Metab Syndr. 15(2):513-518.

13. DeFronzo R, Fleming A, Chen K, Bicsak TA. (2016) Metformin-associated lactic acidosis: current perspectives on causes and risk. Metab Clin Exp. 65: 20-29.

14. Cameron AR, Morrison VL, Levin D, et al. (2016) Antiinflammatory effects of metformin irrespective of diabetes status. Circ Res. 119: 652-665.

15. Kaneto H, Kimura T, Obata A, Shimoda M, Kaku K. (2021) Multifaceted Mechanisms of Action of Metformin Which Have Been Unraveled One after Another in the Long History. Int $\mathbf{J}$ Mol Sci. 22(5):2596.

16. ClinicalTrials.gov Identifier: NCT04510194. (2021) Outpatient treatment of COVID-19 with Metformin. 
This work is licensed under Creative Commons Attribution 4.0 License

To Submit Your Article Click Here: Submit Manuscript

DOI: $10.31579 / 2692-9759 / 072$
Ready to submit your research? Choose Auctores and benefit from:

* fast, convenient online submission

* rigorous peer review by experienced research in your field

* rapid publication on acceptance

* authors retain copyrights

* unique DOI for all articles

* immediate, unrestricted online access

At Auctores, research is always in progress.

Learn more www.auctoresonline.org/journals/endocrinology-anddisorders 